\title{
Induction of ventricular fibrillation predicts sudden death in patients treated with amiodarone because of ventricular tachyarrhythmias after a myocardial infarction
}

Luz-Maria Rodriguez, Eduardo B Sternick, Joep L R M Smeets, Carl Timmermans, Karel den Dulk, Giuseppe Oreto, Hein J J Wellens

\begin{abstract}
Objective-To examine the value of programmed electrical stimulation of the heart in predicting sudden death in patients receiving amiodarone to treat ventricular tachyarrhythmias after myocardial infarction.

Design-Consecutive patients; retrospective study.

Setting-Referral centre for cardiology, academic hospital.

Patients-106 patients with ventricular tachycardia $(n=77)$ or ventricular fibrillation $(n=29)$ late after myocardial infarction.

Interventions-Programmed electrical stimulation was performed while on amiodarone treatment for at least one month.

Measurements and main results-In 80/106 patients either ventricular fibrillation $(n=15)$ or sustained monomorphic ventricular tachycardia $(n=65)$ was induced. After a mean follow up of 50 (SD 40) months (1-144), 11 patients died suddenly and two used their implantable cardioverter defibrillator. By multivariate analysis two predictors for sudden death were found: (1) inducibility of ventricular fibrillation under amiodarone treatment $(P \ll 0 \cdot 001)$, and (2) a left ventricular ejection fraction of $<40 \%(P<0.05)$. The survival rate at one, two, three, and five years was $70 \%, 62 \%, 62 \%$, and $40 \%$ respectively for patients in whom ventricular fibrillation was induced, and $\mathbf{9 8 \%}$, $96 \%, 94 \%, 94 \%$ for patients with induced sustained monomorphic ventricular tachycardia. Where there was no sustained arrhythmia, five year survival was $100 \%$.

Conclusions-In patients receiving amiodarone because of life threatening ventricular arrhythmias after myocardial infarction, inducibility of ventricular fibrillation, but not of sustained monomorphic ventricular tachycardia, indicates a high risk of sudden death.
\end{abstract}

(Heart 1996;75:23-28)

Keywords: ventricular arrhythmias; programmed electrical stimulation; sudden death; myocardial infarction
Studies in the early eighties suggested that programmed electrical stimulation cannot accurately predict recurrences of ventricular tachycardia/ventricular fibrillation during amiodarone treatment. ${ }^{1-4}$ This resulted in the suggestion that high risk patients should be treated with amiodarone empirically without the aid of invasive testing. ${ }^{4}$ More recently, the prognostic value of programmed electrical stimulation in the evaluation of amiodarone treatment for ventricular tachyarrhythmias has again been analysed. ${ }^{5-12}$ Variables helpful in predicting ventricular tachycardia recurrence and sudden death in those studies included persistent ability to induce the clinical arrhythmia, a change in the mode of induction, and modifications of the index arrhythmia while the patient was on amiodarone.

The purpose of our study was to re-examine the value of programmed electrical stimulation of the heart in predicting sudden death in patients receiving amiodarone because of ventricular tachyarrhythmias after myocardial infarction.

\section{Methods}

PATIENTS

The study population consisted of 106 consecutive patients, 92 males and 14 females, with myocardial infarction and sustained ventricular tachyarrhythmias treated with amiodarone, in whom a programmed electrical stimulation study was performed while on amiodarone. Clinical and angiographic data are listed in table 1 .

Evaluation of these patients on admission included clinical history, physical examination, 12-lead electrocardiogram, long term electrocardiographic monitoring, exercise testing, left ventricular ejection fraction measurement, and programmed electrical stimulation while off antiarrhythmic drugs (14 patients) and while on amiodarone in 106 patients. Antiarrhythmic drugs given previously had been empirically selected (not by serial drug testing).

\section{AMIODARONE THERAPY}

Amiodarone was given after previous antiarrhythmic drug treatment had failed in all but 14 patients. In 86 patients failure was due to recurrent spontaneous ventricular arrhythmias and in six because of side effects of the antiar- 
Table 1 Clinical data from the 106 patients studied

\begin{tabular}{ll}
\hline Age (years) (SD, range) & $60(9)(38-84)$ \\
Sex (male/female) & $92 / 14$ \\
Type of arrhythmia & \\
Sustained monomorphic VT & 77 \\
Ventricular fibrillation & 29 \\
Symptoms during arrhythmia & 43 \\
Dizziness & 28 \\
Syncope & 35 \\
Cardiac arrest & 39 \\
Number of previous antiarrhythmic drugs & 45 \\
1 & 8 \\
2 & \\
$>3$ & 54 \\
Myocardial infarction location & 34 \\
Anterior & 18 \\
Inferior & \\
Multiple & 34 \\
No of involved coronary arteries & 35 \\
$>50 \%$ in diameter & 34 \\
1 & $33(10)(15-61)$ \\
3 & 79 \\
Left ventricular ejection fraction (mean \%) (SD, range) & 27 \\
Left ventricular ejection fraction (\%) & 55 \\
< 40\% $40 \%$ & Left ventricular aneurysm
\end{tabular}

VT, ventricular tachycardia. expressed as the mean (SD). $P$ values of $<0.05$ were considered as significant.

\section{Results}

\section{ELECTROPHYSIOLOGICAL STUDY ON}

AMIODARONE

Sustained ventricular arrhythmias were induced in 80 out of 106 patients (75\%). Sustained monomorphic ventricular tachycardia was induced in 65 patients and ventricular fibrillation in 15 . The remaining 26 patients had no sustained ventricular arrhythmia induced.

\section{Type of arrhythmia}

The clinical ventricular arrhythmia (ventricular tachycardia or ventricular fibrillation) was reproduced during the electrophysiological study in 41 patients. A single type of sustained monomorphic ventricular tachycardia, not clinically documented, was induced in 19 patients. More than one type of sustained monomorphic ventricular tachycardia, also not clinically documented, was induced in the remaining 20 patients.

\section{Mode of induction}

Sustained monomorphic ventricular tachycardia and ventricular fibrillation was induced by one ventricular premature beat in nine and two patients respectively. Forty eight patients had monomorphic ventricular tachycardia and eight had ventricular fibrillation induced by two ventricular premature beats. Eight patients with sustained monomorphic ventricular tachycardia and five patients with ventricular fibrillation required three ventricular premature beats for initiation of the arrhythmia.

\section{FOLLOW UP}

\section{Arrhythmic events}

During a mean follow up of 50 (40) months (range 1-144), 29 patients died (27\%). Eleven of the deaths were sudden $(10 \%)$. Two patients in whom a defibrillator was implanted and who received appropriate shocks for haemodynamically poorly tolerated ventricular arrhythmias, as documented by ventricular interval measurements (ventricular rates of 200 to $230 / \mathrm{min}$ ), were added to the sudden death group. Therefore the total sudden death group consisted of 13 patients (11 with sudden death, two needing a defibrillator shock).

Sudden death occurred in (1) nine patients in whom ventricular fibrillation was induced (six with sustained monomorphic ventricular tachycardia and three with ventricular fibrillation as their index arrhythmia); (2) in three patients of the induced sustained monomorphic ventricular tachycardia group; and (3) in one patient in whom no sustained arrhythmia was induced. The last four patients had sustained monomorphic ventricular tachycardia as their index arrhythmia. Twelve patients had cardiac death (12\%), pump failure occurred in nine patients, and a new myocardial infarction in three. Six patients had a non-cardiac death $(6 \%)$. non-sudden cardiac death and com between groups using the Wilcoxon and log rank statistic. Quantitative variables were tested by Student's $t$ test and nominal findings by the $\chi^{2}$ test. Continuous variables are 
Table 2 Relation between the clinical presentation during the index arrhythmia and outcome

\begin{tabular}{|c|c|c|c|c|c|c|c|c|c|}
\hline \multirow[b]{3}{*}{ Index arrhythmia } & \multicolumn{9}{|c|}{ Clinical presentation } \\
\hline & \multicolumn{3}{|c|}{ Dizziness $(n=43)$} & \multicolumn{3}{|c|}{ Syncope $(n=28)$} & \multicolumn{3}{|c|}{ Cardiac arrest $(n=6)$} \\
\hline & $S D$ & $C D$ & $N C D$ & $S D$ & $C D$ & $N C D$ & $S D$ & $C D$ & $N C D$ \\
\hline \multirow[t]{3}{*}{ SMVT } & 3 & 5 & 3 & 3 & 1 & 1 & 1 & 4 & 0 \\
\hline & & & NS & & & $P=0$ & & & \\
\hline & & & & & $\mathbf{P}=$ & & & & \\
\hline VF & - & & & - & & & 6 & 2 & 2 \\
\hline
\end{tabular}

No statistic significance for sudden death between groups. No statistic significance for sudden death between groups.
CD, cardiac death; NCD, non-cardiac death; SD, sudden death; SMVT, sustained monomorphic ventricular tachycardia; VF,
ventricular fibrillation.

Twenty eight patients had syncope during their clinical episode of sustained monomorphic ventricular tachycardia. Three of these patients died from sudden death, one from non-sudden cardiac death, and one from noncardiac death

Comparison between the clinical presentation (dizziness, syncope, or cardiac arrest) during the index arrhythmia (sustained monomorphic ventricular tachycardia/ventricular fibrillation) and outcome is shown in table 2.

The occurrence of sudden death in patients with sustained monomorphic ventricular tachycardia was not related to the clinical presentation. Overall, cardiac death occurred more often in patients with a sustained monomorphic ventricular tachycardia who presented with cardiac arrest than in those presenting with only dizziness or syncope, and sudden death was seen more often in patients with ventricular fibrillation than in patients suffering from sustained monomorphic ventricular tachycardia ( $20 \% v 9 \%)$.

The variables shown in table 3 were first screened by univariate analysis for differences between patients with and without sudden death. Thereafter the same variables were studied in a multivariate model. Inducibility of ventricular fibrillation while on amiodarone treatment for at least one month was the only variable in the univariate model with statistical significance.

In patients with sustained monomorphic ventricular tachycardia as the presenting arrhythmia less ventricular fibrillation was induced and there was a lower incidence of sudden death $(P<0.001)$. Six out of 11 patients $(54 \%)$ in whom ventricular fibrillation was induced on amiodarone treatment and in whom the same ventricular arrhythmia was the index arrhythmia died suddenly.

Table 3 Results of univariate and multivariate analysis: correlation with sudden death

\begin{tabular}{lll}
\hline & Univariate & Multivariate \\
\hline Age & 0.70 & 0.75 \\
Index arrhythmia SMVT/VF & 0.20 & 0.09 \\
Time interval, myocardial infarction to & 0.77 & 0.33 \\
$\quad$ first arrhythmic event $\leqslant$ months & 0.74 & 0.07 \\
Cardiac arrest during the index arrhythmia & 0.44 & 0.70 \\
Myocardial infarction location (anterior & 0.76 & 0.75 \\
$\quad$ inferior) & 0.80 & 0.05 \\
Multiple myocardial infarctions & 0.21 & 0.80 \\
Left ventricular ejection fraction < 40\% & 0.48 & 0.25 \\
Left ventricular aneurysm & 0.19 & $<0.0001$ \\
Multivessel disease & $<0.001$ & \\
Inducibility of VF v SMVT on amiodarone & & \\
\hline treatment & &
\end{tabular}

VF, ventricular fibrillation; SMVT, sustained monomorphic ventricular tachycardia.
Stepwise logistic regression analysis of the clinical, haemodynamic, and electrophysiological data showed that inducibility of ventricular fibrillation $(P<0.001)$ and a low left ventricular ejection fraction $(<40 \%)(P<0.05)$ were independent predictors for sudden death. Syncope or cardiac arrest during the index arrhythmia, and ventricular fibrillation as the clinical arrhythmia were found to be of borderline statistical significance $(P=0.07$ and 0.09 respectively). Of importance was the finding that inducibility of sustained monomorphic ventricular tachycardia while on amiodarone was not a predictor for sudden death.

The outcome of the population according to their index arrhythmia is shown in table 4 .

Actuarial curves for sudden death for patients with induced ventricular fibrillation, sustained monomorphic ventricular tachycardia, and no sustained arrhythmias were constructed. The survival rate at one, two, three, and five years was $70 \%, 62 \%, 62 \%$, and $40 \%$ in the induced ventricular fibrillation patients group, and $98 \%, 96 \%, 94 \%$, and $94 \%$ in the induced sustained monomorphic ventricular tachycardia patients group respectively. Five year survival was $100 \%$ in the group with no sustained arrhythmia (Wilcoxon, $P \ll 0.001$; Log rank $P \ll 0.001$ ) (fig 1 ). The positive predictive value, specificity, and sensitivity

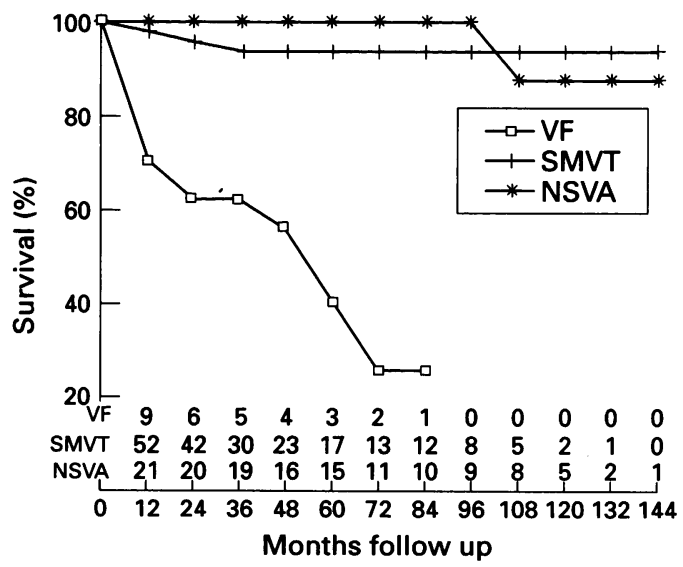

Figure 1 Survival curves (sudden cardiac death) in relation to the arrhythmia induced during amiodarone treatment. NSVA, no sustained ventricular arrhythmias; SMVT, sustained monomorphic ventricular tachycardia; $V F$, ventricular fibrillation. The numbers at the bottom of the graph are the numbers of patients who remained available for analysis at each year during the follow up period. 
Table 4 Outcome of 106 patients with SMVT/VF after myocardial infarction treated with amiodarone. Values are numbers of patients

\begin{tabular}{|c|c|c|c|c|c|c|}
\hline \multirow[b]{3}{*}{ Induced arrhythmia: } & \multicolumn{6}{|l|}{ Index arrhythmia } \\
\hline & \multicolumn{3}{|l|}{$S M V T(n=77)$} & \multicolumn{3}{|l|}{$V F(n=29)$} \\
\hline & $S M V T(n=53)$ & $V F(n=4)$ & $N I(n=20)$ & $S M V T(n=12)$ & $V F(n=11)$ & $N I(n=6)$ \\
\hline \multicolumn{7}{|l|}{ Outcome } \\
\hline Sudden death & 3 & 3 & 1 & 0 & 6 & 0 \\
\hline Cardiac death & 7 & 1 & 2 & 0 & 2 & 0 \\
\hline Non-cardiac death & 0 & 0 & 2 & 2 & 2 & 0 \\
\hline Alive & 43 & 0 & 15 & 10 & 1 & 6 \\
\hline
\end{tabular}

MI, myocardial infarction; NI, non-inducible; SMVT, sustained monomorphic ventricular tachycardia; VF, ventricular fibrillation.

Figure 2 Survival curves (non-sudden cardiac death) in relation to the arrhythmia induced during amiodarone treatment. NSVA, no sustained ventricular arrhythmias, SMVT, sustained monomorphic ventricular tachycardia; VF, ventricular fibrillation. The numbers at the bottom of the graph are the numbers of patients who remained available for analysis at each year during the follow up period.

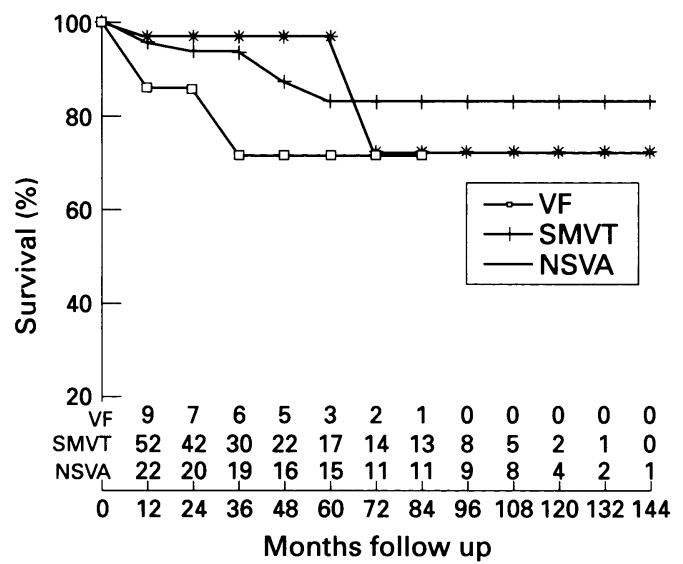

for the inducibility of ventricular fibrillation on amiodarone treatment for at least one month were $60 \%, 94 \%$, and $69 \%$ respectively. Both ventricular fibrillation as index arrhythmia and the left ventricular ejection fraction of $<40 \%$ had a low positive predictive value (six out of 29 [21\%] and 12 out of 79 [15\%], respectively).

When non-sudden cardiac death was used as the end point, no significant differences were found between the three groups (induced ventricular fibrillation [three out of 15 patients], induced sustained monomorphic ventricular tachycardia [seven out of 65

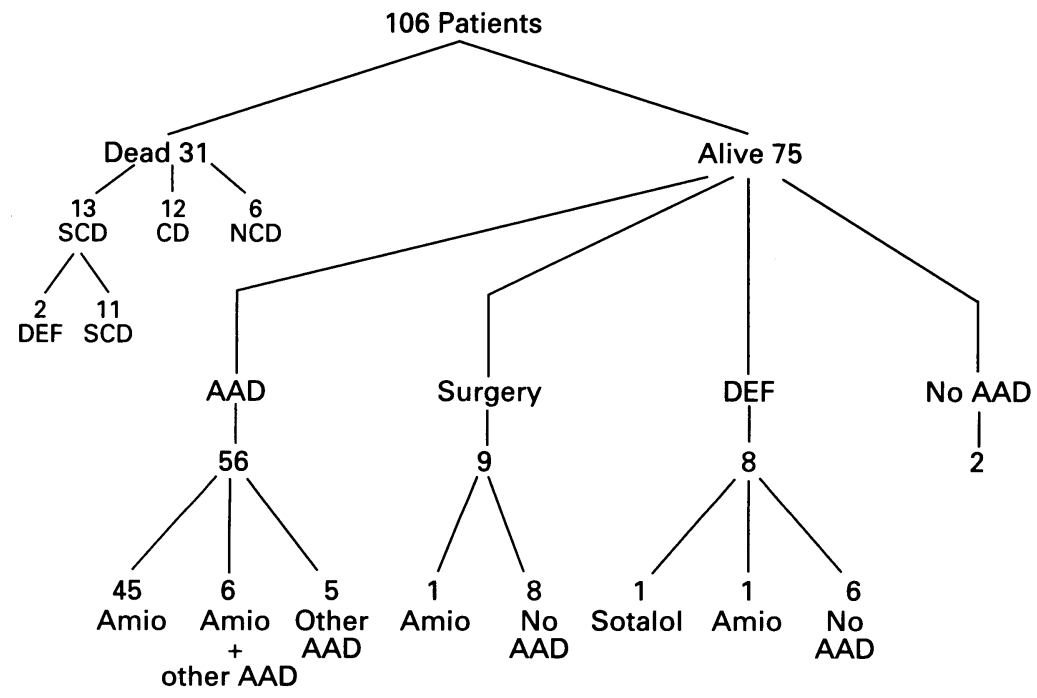

Figure 3 Flow chart showing the follow up of the patients and their current treatment. $A A D$, antiarrhythmic drug; Amio, amiodarone; $C D$, non-sudden cardiac death; DEF, defibrillator; NCD, non-cardiac death; SCD, sudden cardiac death. patients], and no sustained ventricular arrhythmias [two out of 26 patients]). The survival rate from non-sudden cardiac death at one, three, and five years was $85 \%, 71 \%$, and $71 \%$ in the induced ventricular fibrillation group, $95 \%, 93 \%$, and $83 \%$ in the sustained monomorphic ventricular tachycardia group, and $96 \%, 96 \%$, and $96 \%$ in the group with no sustained ventricular arrhythmias, respectively (fig 2).

Non-fatal recurrent ventricular tachycardia was observed in 49 patients $(46 \%)$. Forty two patients had sustained monomorphic ventricular tachycardia and seven had ventricular fibrillation as their index arrhythmia.

\section{Surgery}

During follow up, 18 patients underwent coronary artery bypass surgery because of new ischaemia (13 patients from the sustained monomorphic ventricular tachycardia and five from the ventricular fibrillation index arrhythmia group). Twelve patients had arrhythmia surgery because of ventricular tachycardia recurrences (all were from the sustained monomorphic ventricular tachycardia index arrhythmia group). Three underwent aneurysmectomy alone (one patient died during surgery), five had endocardial resection and aneurysmectomy, and four underwent cryoablation and aneurysmectomy (two patients died, one from chronic lung disease and the other one from pump failure after surgery). One patient who underwent arrhythmia surgery is still on amiodarone.

\section{Side effects}

Side effects were observed in seven out of 106 patients $(7 \%)$. Severe peripheral neuropathy requiring drug discontinuation occurred in one patient. Moderate toxicity was observed in six patients and included visual disturbances in one and hypothyroidism in one. Both patients were continued on amiodarone on a dose of $100 \mathrm{mg}$ daily. Symptomatic AV block either in the AV node $(n=2)$ or distal to the AV node $(n=2)$ necessitated permanent cardiac pacing in four patients.

Current treatment (fig 3)

At the time of writing, 56 of the 75 patients who were still alive were receiving antiarrhythmic drugs. Forty five patients were on amiodarone alone. Six patients were taking amiodarone in combination with another antiarrhythmic drug (amiodarone and fle- 
cainide, $\mathrm{n}=1$; amiodarone and propafenone, $\mathrm{n}=4$; amiodarone and mexitil, $\mathrm{n}=1$ ). Antiarrhythmic drugs other than amiodarone were being used by five patients. This included d-sotalol $(n=1)$, sotalol $(n=1)$, and $\beta$ blockers $(n=3)$. Two patients were without antiarrhythmic drug treatment.

A programmable cardioverter defibrillator was implanted in nine patients because of spontaneously recurring, haemodynamically poorly tolerated ventricular tachyarrhythmias in spite of amiodarone treatment (five patients from the sustained monomorphic ventricular tachycardia and four from the ventricular fibrillation group). One of these patients died postoperatively from sepsis. Two patients had used their defibrillator (Medtronic 7217B) because of ventricular fibrillation in one and fast, haemodynamically poorly tolerated ventricular tachycardia in the other. One patient had documentation of slow ventricular tachycardias not requiring use of the device. In the remaining five patients no spontaneous ventricular tachyarrhythmias requiring electrical treatment had occurred. Two of eight living patients were on antiarrhythmic drugs, one on amiodarone and one on $d$-sotalol.

\section{Discussion}

Our study suggests that programmed electrical stimulation of the heart in patients treated with amiodarone because of sustained monomorphic ventricular tachycardia or ventricular fibrillation after a myocardial infarction allows identification of those patients who are at high risk of dying suddenly.

Inducibility of ventricular fibrillation under amiodarone treatment for at least one month was the strongest predictor of sudden death by univariate and multivariate analysis. A low left ventricular ejection fraction $(<40 \%)$ was also an independent predictor of sudden death. Syncope or cardiac arrest during the index arrhythmia and the index arrhythmia itself (sustained monomorphic ventricular tachycardia/ventricular fibrillation) showed a trend in the multivariate analysis.

Sudden death occurred in $60 \%$ of patients in whom ventricular fibrillation was induced on amiodarone treatment and in $21 \%$ of patients in whom ventricular fibrillation was the index arrhythmia.

In patients with sustained monomorphic ventricular tachycardia as their index arrhythmia ventricular fibrillation was less often induced (5\%) and there was a lower incidence of sudden death (9\%). Induction of a sustained monomorphic ventricular tachycardia or failure to induce a sustained ventricular arrhythmia did not identify patients prone to develop sudden death.

The positive predictive value of induced ventricular fibrillation was better $(60 \%)$ than the positive predictive value of either clinical ventricular fibrillation $(21 \%)$ or a left ventricular ejection fraction of $<40 \%(15 \%)$.

There were no significant differences in the incidence of non-sudden cardiac death between the three groups.
REVIEW OF PUBLISHED REPORTS

The value of programmed electrical stimulation in assessing the efficacy of amiodarone treatment in patients with life threatening arrhythmias is still controversial. Some investigators ${ }^{1-4}$ have reported a poor predictive value of programmed electrical stimulation in patients taking amiodarone, whereas others ${ }^{5-7}$ consider it predictive.

McGovern $e t a l^{6}$ found two significant independent predictors of recurrent arrhythmias: persistence of inducibility of ventricular tachycardia during electrophysiological testing, and a lowered left ventricular ejection fraction. In their study the type of induced ventricular tachycardia (non-sustained or sustained) was not mentioned. Also, fatal (sudden death) and non-fatal events (recurrent ventricular tachycardia) were analysed together.

Klein $e t a l^{8}$ found that easier induction of ventricular tachycardia during amiodarone treatment versus control was highly predictive of arrhythmia recurrence. In their paper sudden death and recurrent ventricular tachycardia were also not differentiated.

Kadish et $a l^{9}$ looked for predictors of recurrent ventricular tachycardia and sudden death. No predictor of recurrent ventricular tachycardia was found. However, they did find predictors of cardiac arrest or sudden death. These included haemodynamic instability of the arrhythmia induced on electrophysiological testing during amiodarone treatment, younger age, low left ventricular ejection fraction, the presence of a left ventricular aneurysm, and a poorly tolerated rhythm at clinical presentation. Survival at one and three years of patients with poorly tolerated arrhythmias induced at electrophysiological study during amiodarone treatment were similar to our results $(75 \% v 70 \%$, and $70 \% v 62 \%$ respectively).

\section{LIMITATIONS OF OUR STUDY}

The optimal time to assess the role of the electrophysiological study in patients treated with amiodarone because of life threatening arrhythmias with coronary artery disease is still controversial. The pharmacokinetic profile of amiodarone is unusual and not fully understood, making it difficult to determine when steady state is achieved with this agent. ${ }^{17} 18$

The value of early electrophysiological studies in patients taking amiodarone for 10 to 14 days in predicting outcome from ventricular tachyarrhythmias in coronary artery disease has been reported by Manolis et al. ${ }^{11}$ However, since clinical practice suggests that it may take several weeks of amiodarone loading before full clinical efficacy can be established, ${ }^{17} 18$ we selected a period of one month of amiodarone treatment to evaluate the role of the electrophysiological study in predicting outcome.

Our study is a retrospective one, from a tertiary referral centre with a limited number of patients. To be admitted to the study the patient had to be on amiodarone for at least one month, thereby excluding patients dying early after the onset of their arrhythmia. This 
may explain why, in contrast to previous studies, ${ }^{19} 20$ we did not find that clinical variables such as (1) a time interval of $<2$ months between myocardial infarction and the first episode of sustained ventricular arrhythmia, (2) syncope during the presenting arrhythmia, (3) presence of multiple myocardial infarctions, or (4) location of myocardial infarction (anterior $v$ inferior) were of value in predicting risk of sudden death during follow up.

Another limitation of our study was that only 14 patients underwent baseline electrophysiological examination. This feature is explained by the fact that the majority of our patients were already on amiodarone treatment when referred to our hospital.

\section{CONCLUSIONS}

Our retrospective study suggests that in patients receiving amiodarone because of life threatening ventricular arrhythmias after myocardial infarction, the inducibility of ventricular fibrillation, but not of a sustained monomorphic ventricular tachycardia, indicates a high risk of dying suddenly. We believe that this finding should now be evaluated prospectively. It might be of help in selecting patients for non-pharmacological treatment.

1 Heger J, Prystowsky EN, Jackman WM, Nacarelli GV, Warfel KA, Rinkenberger RL, et al. Amiodarone: clinical efficacy and electrophysiology during long-term therapy for reurre lectrophys lation. $N$ Engl ₹ Med 1981;305:539-45.

2 Hamer AW, Finerman WB, Peter T, Mandel WJ. Disparity between the clinical and electrophysiologic effects of amiodarone in the treatment of recurrent ventricular amiodarone in the treatment of recurrent ventric

3 Waxman HL, Groh WC, Marchlinski FE, Buxton AE Sadowski LM, Horowitz LN, et al. Amiodarone for conSadowski LM, Horowitz LN, et al. Amiodarone for con-
trol of sustained ventricular tachyarrhythmias. Clinical trol of sustained ventricular tachyarrhythmias. Clinical and electrophysiolo

4 Nademanee $K$, Hendrickson JA, Jannan R, Singh BH Antiarrhythmic efficacy and electrophysiologic actions of amiodarone in patients with life-threatening ventricula arrhythmias: potent suppression of spontaneously occurring tachyarrhythmias versus inconsistent abolition of induced ventricular tachycardia. Am Heart $f 1982 ; 103$. 950-9.
5 Horowitz LN, Greenspan AM, Spielman SR, Webb CR, Morganroth J, Rotmensch $\mathrm{H}$, et al. Usefulness of electrophysiologic testing in evaluation of amiodarone therapy for sustained ventricular tachyarrhythmias associated with coronary artery disease. Am $\mathcal{f}$ Cardiol 1985;55: 367-71.

6 McGovern B, Garan H, Malacoff RF, DiMarco JP, Garant $\mathrm{G}$, Sellers $\mathrm{D}$, et al. Long-term clinical outcome of ventricular tachycardia or fibrillation treated with amiodarone. Am $\mathcal{F}$ Cardiol 1984;53:1558-63.

7 Nacarelli GV, Fineberg NS, Zipes DP, Heger JJ, Duncan G, Prystowsky EN. Amiodarone: risk factors for recurrences of symptomatic ventricular tachycardia identified at electrophysiologic study. $¥ \mathrm{Am}$ Coll Cardiol 1985;68: a1 $81-21$.

8 Klein LS, Fineberg N, Heger J, Miles WM, Kammerling $\mathrm{JM}$, Chang M-S, et al. Prospective evaluation of a descriminant function for prediction of recurrent ventricular tachycardia or ventricular fibrillation in coronary artery disease patients receiving amiodarone and having inducible ventricular tachycardia at electrophysiologic study. Am f Cardiol 1988;61:1024-30.

9 Kadish AH, Buxton AE, Waxman HL, Flores B, Josephson ME, Marchlinski FE. Usefulness of electrophysiologic study to determine the clinical tolerance of arrhythmia recurrences during amiodarone therapy. $\mathcal{f} \mathrm{Am} \mathrm{Col}$ Cardiol 1987;10:90-6.

$10 \mathrm{Zhu}$ J, Haines DE, Lerman BB, DiMarco JP. Predictors of efficacy of amiodarone and characteristics of recurrence of arrhythmia in patients with sustained ventricular of artich 76:802-9.

11 Manolis AS, Urichio F, Estes ME. Prognostic value of early electrophysiologic studies for ventricular tachycardia electrophysiologic studies for ventricular tachycardia recurrence in patients with coronary artery disease

$12 \mathrm{Kim}$ SG, Felder SD, Figura I, Johnston DR, Mercado AD, Fisher JD. Prognostic value of changes in the mode of ventricular tachycardia induction during therapy with amiodarone and class $1 \mathrm{~A}$ antiarrhythmic agent. $\mathrm{Am} \mathfrak{f}$ Cardiol 1987;59:1314-8.

13 Brugada P, Wellens HJJ. Programmed electrical stimulation of the human heart. In: Josephson ME, Wellens HJ, eds. Tachycardias; mechanism, diagnosis and treatment. Philadelphia: Lea and Febiger, 1984;61-89.

14 Yusuf S, Garg R, Zucker D. Analysis by intention-to-treat principle in randomized trials and databases. $P A C E$ 1991;14:2078-82.

15 The SAS software. In: PHREG procedure, 1990:435-79.

16 Cox DR. Regression models and life tables. I Stat Soc (b) 1972;34:187-220.

17 Holt DW, Tucker GT, Jacson PR, Storey GCA Amiodarone pharmacokinetics. Am Heart $\mathcal{f}$ 1983;106: 840.

18 Haffajee CI, Love JC, Canada AT, Lesko LJ, Askourian G Alpert JS. Clinical pharmacokinetics and efficacy of amiodarone for refractory tachyarrhythmias. Circulation amiodarone for refr.

19 Brugada P, Talajic M, Smeets J, Mullenders R, Wellens HJ. Risk stratification of patients with ventricular tachycardia or ventricular fibrillation after myocardial infarction. The value of the clinical history. Eur Heart $\mathcal{F} 1989$, 10:747-52.

20 Willems AR, Tijssen JGP, Van Capelle FJL, et al. Determinants of prognosis in symptomatic ventricular tachycardia or ventricular fibrillation late after myocardial infarction. $\mathcal{F}$ Am Coll Cardiol 1990;16:521-30. 